\title{
Monocyte to Lymphocyte Ratio in Peripheral Blood of Tuberculous Meningitis with HIV Patients in Tertiary Hospital in West Java
}

\author{
Ridha Amalia,, ${ }^{1}$ Dewi Kartika Turbawaty, ${ }^{2}$ Adhi Kristianto Sugianli, ${ }^{2}$ Siti Aminah ${ }^{3}$ \\ ${ }^{1}$ Faculty of Medicine Universitas Padjadjaran, Bandung, Indonesia \\ ${ }^{2}$ Department of Clinical Pathology, Faculty of Medicine, Universitas Padjadjaran-Dr. Hasan Sadikin General \\ Hospital, Bandung \\ ${ }^{3}$ Department of Neurology, Faculty of Medicine, UniversitasPadjadjaran-Dr. Hasan Sadikin General Hospital, \\ Bandung
}

\begin{tabular}{|c|c|}
\hline \multirow[t]{4}{*}{ Abstract } & $\begin{array}{l}\text { Objective: To describe peripheral blood monocyte to lymphocyte (ML) ratio } \\
\text { in tuberculous meningitis (TBM) patients with human immunodeficiency } \\
\text { virus (HIV) infection in Dr. Hasan Sadikin General Hospital Bandung as a } \\
\text { tertiary hospital in West Java Province, Indonesia. }\end{array}$ \\
\hline & $\begin{array}{l}\text { Methods: A cross-sectional study with retrospective data approach was done } \\
\text { in Dr. Hasan Sadikin General Hospital, Bandung. Data were collected from } \\
\text { TBM patients who were hospitalized in Dr. Hasan Sadikin General Hospital, } \\
\text { Bandung in the period of } 2014-2016 \text {. The sample size was determined } \\
\text { using total sampling method. General data, clinical manifestation data, and } \\
\text { laboratory finding data were collected. The ML ratio was collected and } \\
\text { presented into three groups of percentile }\left(25^{\text {th }}, 25-75^{\text {th }} \text { and } 75^{\text {th }}\right) \text { based on } \\
\text { the pre-defined cut-off. }\end{array}$ \\
\hline & $\begin{array}{l}\text { Results: Six subjects }(40 \%) \text { had an ML ratio of } \leq 0.47 \text { and } 3 \text { subjects had an } \\
\text { ML ratio of } \geq 1.22 \text { in TBM with HIV population. Among TBM with HIV-negative } \\
\text { population, there were } 16 \text { subjects had ML ratio } \leq 0.47 \text {, and } 19 \text { subjects had } \\
\text { ML ratio } \geq 1.22 \text {. }\end{array}$ \\
\hline & $\begin{array}{l}\text { Conclusions: The ML ratio in TBM with HIV infection has become additional } \\
\text { tool for detecting the development of TBM with HIV-infected, as well as TBM }\end{array}$ \\
\hline $\begin{array}{l}\text { Received: } \\
\text { December 6, } 2017\end{array}$ & $\begin{array}{l}\text { with non-HIV-infected. Therefore, it could help directing the patients with } \\
\text { clinical suspicious of TBM into the diagnosis algorithm and treatment of TBM, } \\
\text { to have better outcome. }\end{array}$ \\
\hline $\begin{array}{l}\text { Revised: } \\
\text { March 5, } 2018\end{array}$ & $\begin{array}{l}\text { Keywords: Human immunodeficiency virus infection, lymphocyte, monocyte, } \\
\text { monocyte to lymphocyte ratio, tuberculous meningitis }\end{array}$ \\
\hline $\begin{array}{l}\text { Accepted: } \\
\text { March 8, } 2018\end{array}$ & $\begin{array}{l}\text { pISSN: 2302-1381; eISSN: 2338-4506; http://doi.org/10.15850/ijihs.v6n1.1140 } \\
\text { IJIHS. 2018;6(1):42-7 }\end{array}$ \\
\hline
\end{tabular}

\section{Introduction}

Humanimmunodeficiencyvirus(HIV) infection is one of the main global health problems and becomes a risk factor for tuberculosis. ${ }^{1,2}$ The characteristics of immunological effect of HIV is the CD 4 cell count. ${ }^{2}$ People with HIV infection also have a higher risk to get all types of extra pulmonary tuberculosis, including tuberculous

\section{Correspondence:}

Ridha Amalia, Faculty of Medicine,

Universitas Padjadjaran

Jl. Raya Bandung-Sumedang KM 21 Jatinangor,

Indonesia

e-mail: ridhaamalia11@gmail.com meningitis (TBM). ${ }^{3}$ Tuberculous meningitis is the most common cause of meningitis in HIV-infected people along with cryptococcus meningitis. ${ }^{4,5}$ Meningitis is considered as the worst manifestation of tuberculosis. ${ }^{6,7}$ Their combined appearance makes diagnosis and treatment challenging due to the unspecific and similar clinical manifestations to other causes of chronic meningoencephalitis and also because it is necessary to provide treatment for both infections and their complication. ${ }^{8}$

Knowledge on hematological manifestation of Mycobacterium tuberculosis (Mtb) infection is important to provide knowledge on the pathogenesis of the disease. ${ }^{9}$ Myeloid-specific 
cells have been known as the host cells for Mtb growth and lymphoid cells are known as the main effector cells in tuberculosis immunity. ${ }^{9,10}$ The main role of monocytes and lymphocytes in inducing that immune response makes its level in Monocytes to Lymphocyte (ML) ratio is expected to be able to reflect the person's immune condition toward the infection. ${ }^{9}$

Study on patients with active tuberculosis showed that a higher median absolute number of monocytes and a lower median absolute number of lymphocytes were observed among these patients when compared to the healthy controls. ${ }^{9}$ Hence, ML ratio could be used to differentiate patients with active tuberculosis from healthy people. ${ }^{9}$ A retrospective study on patients with active tuberculosis showed that higher or lower ML ratio based on a predefined cut-off could be a significant predictor for active tuberculosis. ${ }^{9}$ Patients with extra pulmonary tuberculosis had an ML ratio as high as almost 2.5 times higher compared to the normal group. ${ }^{9}$ Many studies showed that the ML ratio could be used to stratify tuberculosis risks and the involvement of hematopoietic stemcell in tuberculosis pathogenesis among HIVinfected people. ${ }^{10}$ Therefore, ML ratio could be used as an independent prognostic marker and a marker of a successful anti-tuberculosis therapy. ${ }^{11}$

Screening and diagnostic testing of TBM in Dr. Hasan Sadikin General Hospital Bandung still face several challenges: complicated TBM examination algorithm, expensive diagnostic tests, low economic status of the patients, lack of education, etc. Due to these difficulties in diagnosing TBM with HIV co-infection, studies related to a simple detection or screening tool for TBM need to be done. ${ }^{5,7}$ The objectives of this study was to describe the ratio of monocytes to lymphocytes in peripheral blood as a predictor for TBM with HIV infection to seek the possibility to use the ratio as an additional tool for the clinician to perform the diagnostic algorithm of TBM.

\section{Methods}

This was a cross-sectional study using TBM retrospective clinical-based data. Population of this study consisted of TBM patients who were hospitalized in Dr Hasan Sadikin General Hospital Bandung during the period of 20142016. The inclusion criteria included patients aged 18 years old or above diagnosed with TBM based on the diagnostic criteria from Marais et $a .^{7}$ and had performed the first laboratory examination (hematology, immunology and cerebrospinal fluid (CSF) examination) after being diagnosed TBM with complete data. Patients with any missing data were excluded from this study.

Patients were divided into 4 groups based on the diagnostic criteria stated by Marais et $a .^{7}$ The criteria were definite tuberculous meningitis, probable tuberculous meningitis, possible tuberculous meningitis, and not tuberculous meningitis. The description on the components of these criteria and the scoring procedure is available elsewhere.

This study used total sampling collection method, of which all members of the population were recruited in the study. All data regarding hospitalized TBM patients were collected from the medical record database of Department of Neurology, Dr. Hasan Sadikin General Hospital Bandung during the period of 2014-2016. Those who fulfilled the inclusion criteria were included in the analysis.

The data collection process was performed in 2 places: Department of Neurology and Medical Record Unit of Dr. Hasan Sadikin General Hospital Bandung. Data that were collected included data on patients' general characteristics, clinical manifestations, and laboratory results. Data collected were also double-checked for correctness with data on routine hematology with differential count and CSF examination from the Department of Clinical Pathology, Dr. Hasan Sadikin General Hospital, Bandung. Data collected were then merged in a prepared database for analysis.

Two hundred and eighty patients met the inclusion criteria of this study. Of these, 195 were excluded due to incomplete data: absent of differential count test, no lumbar puncture was performed, or no HIV testing was done. The flowchart of this study was summarized in the diagram (Fig. 1).

This study was approved by the Health Research Ethic Committee of the Faculty of Medicine, Universitas Padjadjaran and from the Research Education Unit of Dr. Hasan Sadikin General Hospital, Bandung.

Patients' data consisted of name, gender, age, diagnosis, HIV status, result of routine hematological tests and differential count, and results of CSF examination were collected and entered into a pre-prepared database (Excel software, Microsoft Corp.). All data were processed and presented using percentage in a table to describe the gender, age group, clinical manifestation, CSF analysis, and ML ratio. The ML ratios were tabulated based on the HIV status and patient's TBM diagnosis, 


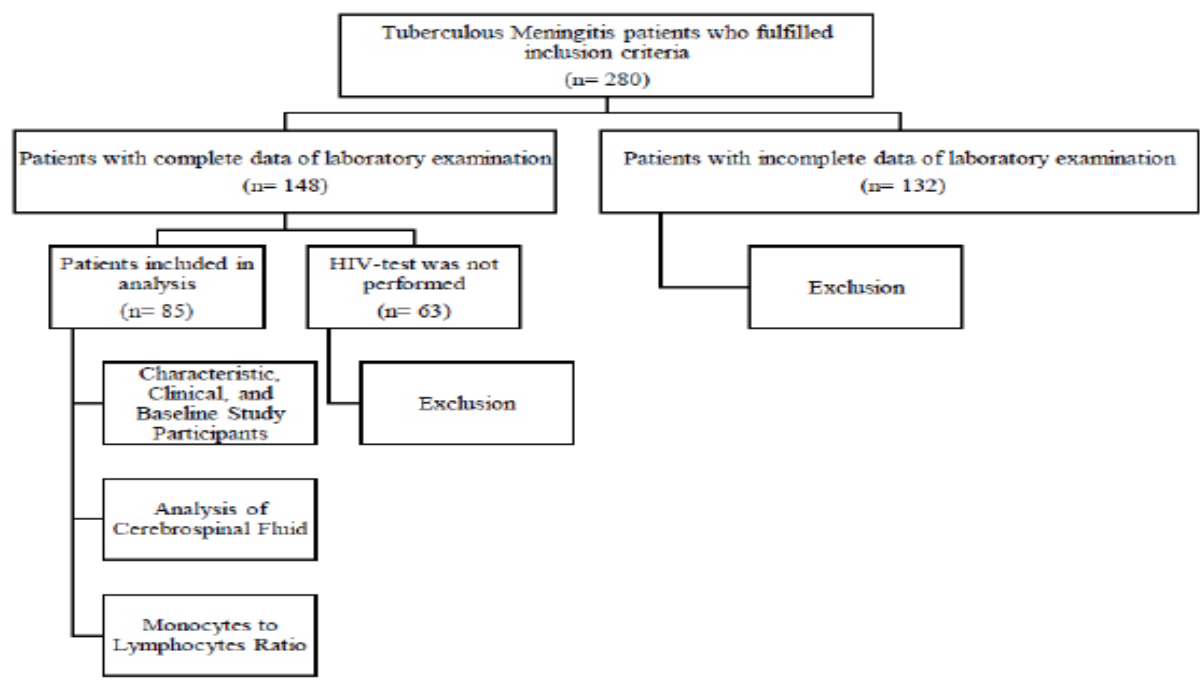

Fig. 1 Screening and Inclusion of Study Subject Flowchart

which were divided into possible, probable, and definite TBM. The ML ratios were then classified and presented in percentiles: (1) $\leq 25^{\text {th }}$ percentile $\mathrm{ML}$ ratio of all patients (ML ratio $\leq 0.47)$, (2) between $25^{\text {th }}-75^{\text {th }}$ percentile $(0.47<\mathrm{ML}$ ratio $<1.22),(3) \geq 75$ th percentile ML ratio of all patients (ML ratio $\geq 1.22$ ). ${ }^{9} \mathrm{~A}$ bar chart was used to present the ML ratio in the population. All the data were analyzed using STATA version 12.0 (Stata Corp., Texas, USA).

\section{Results}

In the population of this study, 15 patients (18\%) were diagnosed as suffering from TBM with HIV- positive and 70 patients (82\%) with HIV negative. Among the TBM with HIV positive population, male patients were more dominant ( $87 \%$ ) as well as the 25-34 years old age group $47 \%$ ). This finding is similar with the finding in a study performed by from Afriandi et al. $^{12}$ in 2009 that described the national AIDS cases were dominated by males (75\%) with $42 \%$ were reported to be IDU-related. Heterosexual and homosexual transmission was $48 \%$ and $3.7 \%$, respectively. Most cases found in this study were classified as probable TBM (63.5\%) in accordance with diagnostic criteria from Marais et al. ${ }^{7}$ This number was followed by possible TBM (34.1\%) and then definite TBM $(2.4 \%)$ categories. The four most commonly identified clinical manifestations were headache, neck stiffness, fever, as well as decreased level of consciousness. Results of CSF examination showed that there was a process of meningeal irritation, which was proven by high number of leukocyte count $\left(>5\right.$ cells $\left./ \mathrm{mm}^{3}\right)$, low CSF-serum glucose ratio $(<50 \%)$, and high level of protein (>50 mg/ $\mathrm{dL}$ ) in most subjects. The median of ML Ratio among HIV positive patients was 0.81 while the HIV negative patients had a ratio of 0.67 (Table 1).

In TBM with HIV positive population, there were 6 subjects who had an ML ratio of 0.47 and 3 with an ML ratio of 1.22 . In TBM with HIV negative population, 16 subjects had an ML ratio of 0.47 and 19 had an ML ratio of 1.22.

\section{Discussion}

Hematological changes could be used as the first hallmark of a disease process as radiological changes and physical signs often appear later. ${ }^{13}$ Monocyte is among the important components of innate immune response that act as a link to the adaptive immune system by presenting antigen to lymphocyte. ${ }^{9}$ Hence, any factor that influences the function or relative number of both cells could potentially affect individual's response toward infection. ${ }^{9}$

Peripheral blood monocyte to lymphocyte ratio could reflects an individual's capacity to mount effective immune responses. ${ }^{9}$ This ratio has been found to correlate with Mycobacterial in vitro growth inhibition and is considered 
Ridha Amalia, Dewi Kartika Turbawaty, et al.

Table 1 Characteristic, Clinical Manifestation, Labolatory Examination of Study Subjects

\begin{tabular}{|c|c|c|}
\hline Variable & HIV Positive (n=15) & HIV Negative $(n=70)$ \\
\hline \multicolumn{3}{|l|}{ Gender } \\
\hline Female & $2(13 \%)$ & 25 (36\%) \\
\hline Male & $13(87 \%)$ & $45(64 \%)$ \\
\hline \multicolumn{3}{|l|}{ Age group, years } \\
\hline $18-24$ & $3(20 \%)$ & $22(31 \%)$ \\
\hline $25-34$ & $7(47 \%)$ & $25(36 \%)$ \\
\hline $35-49$ & $5(33 \%)$ & $15(21 \%)$ \\
\hline$>49$ & $0(0 \%)$ & $8(11 \%)$ \\
\hline \multicolumn{3}{|l|}{ Tuberculous meningitis } \\
\hline Possible & $5(33 \%)$ & $24(34 \%)$ \\
\hline Probable & $9(60 \%)$ & $45(64 \%)$ \\
\hline Definite & $1(7 \%)$ & $1(1 \%)$ \\
\hline \multicolumn{3}{|l|}{ Clinical presentation, $N$} \\
\hline Decrease of level of consciousness & $13(87 \%)$ & $61(87 \%)$ \\
\hline Neck stiffness & $12(80 \%)$ & $65(93 \%)$ \\
\hline Headache & $13(87 \%)$ & $66(94 \%)$ \\
\hline Fever & $12(80 \%)$ & $61(87 \%)$ \\
\hline Vomiting & $5(33 \%)$ & $21(30 \%)$ \\
\hline Seizure & $2(13 \%)$ & $5(7 \%)$ \\
\hline \multicolumn{3}{|l|}{ CSF analysis, $N$} \\
\hline Leukocyte count $>5$ cells $/ \mathrm{mm}^{3}$ & $10(67 \%)$ & $66(94 \%)$ \\
\hline Glucose ratio $<50 \%$ & $13(87 \%)$ & $61(87 \%)$ \\
\hline Protein $>50 \mathrm{mg} / \mathrm{dL}$ & $11(73 \%)$ & $64(91 \%)$ \\
\hline \multicolumn{3}{|l|}{ Hematology cell-count, median (range) } \\
\hline Lymphocyte count, median (range), \% & $13(4-56)$ & $8(2-47)$ \\
\hline Monocyte count, median (range) \% & $8(4-15)$ & $6(1-21)$ \\
\hline Monocyte to lymphocyte ratio, median (range) & $0.81(0.13-2.80)$ & $0.67(0.03-1.62)$ \\
\hline
\end{tabular}

Data presented as n (\%) unless there was another explanation mentioned.

Abbreviation: CSF= cerebrospinal fluid; HIV= human immunodeficiency virus

a risk if found in person with high or low ML ratio, based on the percentile cut-off., ${ }^{9,14}$ It has been reported that Mtb infection could disturb hematopoietic stem cells or may directly infect the bone marrow mesenchymal stem cells. ${ }^{9,15}$ Studies in mice and human have shown that hematopoietic stem cells present a different ratio of myeloid to lymphoid cells they will give rise to. ${ }^{9,16,17}$ Different proportions of myeloid hematopoietic stem cells or lymphoid could lead to the difference of ML ratio in peripheral blood. ${ }^{9}$ Hence, it is probable that Mtb infection could affect hematopoietic stem cells as well as the ML ratio being affected. ${ }^{9}$

Most subjects in this study were found to have lymphopenia. This is similar to a previous study by Okamura et al. ${ }^{18}$ Lymphopenia might occur due to the accumulation of lymphocytes in the infection sites, leading to a decrease in the number of lymphocites in the peripheral blood. ${ }^{11,19}$ For monocytes, most patients have normal or high monocyte count (monocytosis). 


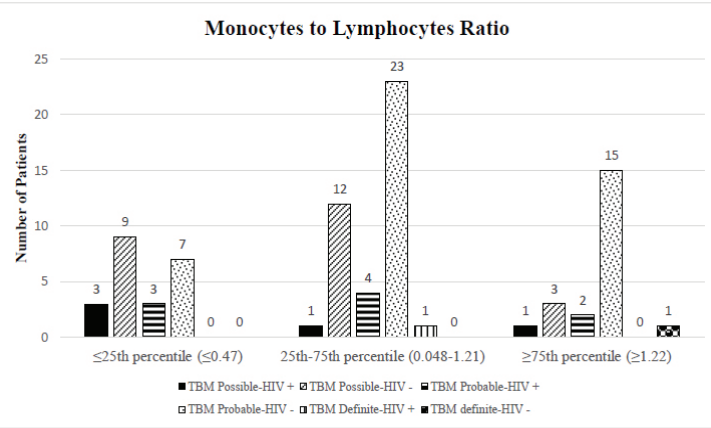

Abbreviation: HIV= human immunodeficiency virus; $\mathrm{TBM}=$ tuberculous meningitis

\section{Fig. 2 Monocyte to Lymphocyte Ratio among Tuberculous Meningitis with- or without Human Immunodeficiency Virus Patients}

Monocytosis might occur because monocyte have a role in phagocytosis of microorganism. ${ }^{20}$ Microorganisms which successfully escape the immune response cause infection and produce chemo attractant substances that will invite other leukocytes and resulting in unopposed production of monocytes. ${ }^{20}$ This creates a different ML ratio in patients when compared with healthy people.

Monocyte to lymphocyte ratio is a simple tools that is expected to reflect the person's immune condition toward the infection. The normal average of ML ratio is $0.3 .{ }^{19}$ The TBM with HIV negative patients in this study had a higher ML ratio. The majority of the subjects have an ML ratio of more than $0.47\left(>25^{\text {th }}\right.$ percentile). This is similar to the finding in a previous study by Wang et al. ${ }^{9}$ with increased ML ratio among active tuberculosis and extra pulmonary tuberculosis patients and a lower or higher ML ratio can predict the presence of active tuberculosis. In TBM with HIV positive a higher ML ratio was also seen while some subjects had a lower ML ratio (less than or equals 0.47 ). This is similar to a previous study with HIV patients as the subjects by Naranbhai et $a .^{10}$ that showed tuberculosis is higher in HIV patients with lower or higher ML ratio. This situation might be explained by immune response toward Mtb. Myeloid-specific cells have been known to have role as host cells for Mtb growth and lymphoid cells as the main effector in TB immunity. ${ }^{9,10}$ Therefore, most of the TBM subjects in this study have higher ML ratio. Within this study, any clear distinguish of ML ratio among each group was not found and further comprehensive study is needed to analyze. Overall, the results of this current study gives evidence for supporting the correlation between immunity and tuberculosis as well as extra pulmonary tuberculosis, as described in previous study. Hence, ML ratio could be used as a tool for detecting active TBM, either with or without HIV infection.

This study has several limitations. This was retrospective study used data from medical record database. Since the diagnosis was made using Marais criteria, there might be a slight difference among clinicians when establishing the TBM diagnosis. Despite the limitations, this study supports the notion that ML ratio is disrupted in TBM population with or without HIV infection as already mentioned in several previous studies. However, a prospective study using dedicated population and sample size need to be done to find the actual correlation between ML ratio and TBM.

This study shows that the ML ratio in TBM patients with HIV infection at Dr. Hasan Sadikin General Hospital, Bandung can be used as an additional tool for detecting the development of TBM in patients with HIV as well as in nonHIV-infected patients. Therefore, it could help to direct the patients with clinical suspicion of TBM into TBM diagnosis algorithm and treatment and to a better disease outcome. The results of this study can also be used to create an evidence based-medicine approach in Dr. Hasan Sadikin General Hospital, Bandung as a teaching hospital and tertiary hospital in West Java, Indonesia.

\section{References}

1. World Health Organization | HIV/AIDS Fact Sheets. 2016.[cited 2017 Aug 17]. Available from: http://www.who.int/mediacentre/ factsheets/fs360/en/.

2. Walker NF, Meintjes G, Wilkinson RJ. HIV-1 and the immune response to TB. Future Virol.

2013;8(1):57-80.

3. Vinnard C, Macgregor RR. Tuberculous meningitis in HIV-infected individuals. Curr HIV/AIDS Rep. 2009;6(3):139-45.

4. Ghosh GC, Sharma B, Gupta BB. CSF ADA determination in early diagnosis of tuberculous 
meningitis in HIV-infected patients. Scientifica [serial on the internet]. 2016 Apr [cited 2017 Jun 10];2016(1):[about 4p.]. Available from: https://www.ncbi.nlm.nih.gov/pmc/articles/ PMC4837278/.

5. Veltman JA, Bristow CC, Klausner JD. Review article meningitis in HIV-positive patients in sub-Saharan Africa: a review. J Int AIDS Soc. 2014;17(1):1-10.

6. Isabel BE, Rogelio HP. Pathogenesis and immune response in tuberculous meningitis. Malays J Med Sci. 2014;21(1):4-10.

7. Marais S, Thwaites G, Schoeman JF, Török ME, Misra UK, Prasad K, et al. Tuberculous meningitis: a uniform case definition for use in clinical research. Lancet Infect Dis. 2010;10(11):803-12.

8. Török ME. Tuberculous meningitis: advances in diagnosis and treatment. $\mathrm{Br}$ Med Bull. 2015;113(1):117-31.

9. Wang J, Yin Y, Wang X, Pei H, Kuai S, Gu L. Ratio of monocytes to lymphocytes in peripheral blood in patients diagnosed with active tuberculosis. Braz J Infect Dis. 2015;19(2):125-31.

10. Naranbhai V, Hill AVS, Abdool Karim SS, Naidoo K, Abdool Karim Q, Warimwe GM, et al. Ratio of monocytes to lymphocytes in peripheral blood identifies adults at risk of incident tuberculosis among HIV-infected adults initiating antiretroviral therapy. J Infect Dis. 2014;209(4):500-9.

11. Iqbal S, Ahmed U, Khan MA. Haematological parameters altered in tuberculosis. Pak J Physiol. 2015;11(1):13-6.

12. Afriandi I, Aditama TY, Mustikawati D, Oktavia M, Alisjahbana B, Riono P. HIV and injecting drug use in Indonesia: epidemiology and national response. Acta Med Indones. 2009;41(1):75-8.

13. Agarwal A, Bhat MS, Kumar A, Shaharyar A, Mishra M, Yadav R. Lymphocyte/monocyte ratio in osteoarticular tuberculosis in children: a haematological biomarker revisited. Trop Doct. 2016;46(2):73-7.

14. Tobin DM, Roca FJ, Oh SF, McFarland R, Vickery TW, Ray JP, et al. Host genotype-specific therapies can optimize the inflammatory response to mycobacterial infections. Cell. 2012;148(3):434-46.

15. Baldridge MT, King KY, Boles NC, Weksberg DC, Goodell MA. Quiescent haematopoietic stem cellsareactivated by IFN- $\gamma$ in response to chronic infection. Nature. 2010;465(7299):793-7.

16. Schroeder T. Hematopoietic stem cell heterogeneity: subtypes, not unpredictable behavior. Cell Stem Cell. 2010;6(3):203-7.

17. Muller-Sieburg CE, Sieburg HB, Bernitz JM, Cattarossi G. Stem cell heterogeneity: Implications for aging and regenerative medicine. Blood. 2012;119(17):3900-7.

18. Okamura K, Nagata N, Wakamatsu K, Yonemoto $\mathrm{K}$, Ikegame S, Kajiki A, et al. Hypoalbuminemia and lymphocytopenia are predictive risk factors for in-hospital mortality in patients with tuberculosis. Intern Med. 2013;52(4):439-44.

19. Muller GL, Davidson DL. The monocytelymphocyte ratio as ameasurement of activity in pulmonary tuberculosis. $\mathrm{N}$ Engl J Med. 1934;211(6):248-52

20. Iqbal S, Ahmed U, Badshah S, Zaidi H. Monocyte lymphocyte ratio as a possible prognostic marker in antituberculous therapy. JRawal Med College. 2014;18(2):178-81. 